\title{
Number of Biological Brothers
}

National Cancer Institute

\section{Source}

National Cancer Institute. Number of Biological Brothers. NCI Thesaurus. Code C135500.

The number of brothers that are biologically related to the subject. 